\title{
Quantum of Thermal Conductance of Nanofilms due to Surface-Phonon Polaritons
}

\author{
Yangyu Guo, ${ }^{1}$ Saeko Tachikawa, ${ }^{1}$ Sebastian Volz, ${ }^{1,2}$ Masahiro Nomura, ${ }^{1}$ and Jose Ordonez-Miranda ${ }^{1,2, *}$ \\ ${ }^{1}$ Institute of Industrial Science, The University of Tokyo, Tokyo 153-8505, Japan \\ ${ }^{2}$ LIMMS, CNRS-IIS UMI 2820, The University of Tokyo, Tokyo 153-8505, Japan
}

(Dated: October 18, 2021)

\begin{abstract}
Based on the Boltzmann transport equation, we demonstrate that the thermal conductance per unit width of a sufficiently thin polar nanofilm supporting the propagation of surface-phonon polaritons along its surfaces is independent of the material properties and is given by $12 z(3) k_{B}^{3} T^{2} / \mathrm{ch}^{2}$, where $k_{B}$ and $h$ are the respective Boltzmann and Planck constants, while $c$ is the light speed in vacuum, $T$ the temperature and $z(3)$ the Riemann zeta function. The huge propagation length of these energy carriers establishes that this quantization holds not only for temperatures much smaller than $1 \mathrm{~K}$, as is the case of electrons and phonons, but also for those comparable to room temperature, which can significantly facilitate its observation and implementation in the thermal management of nanoscale electronics and photonics.
\end{abstract}

PACS numbers: 65.40.-b; 65.60.+a; 65.90.+i

One-dimensional (1D) heat conduction at low temperature has been extensively investigated due to the existence of a quantum of thermal conductance. This quantization corresponds to the heat flux carried by ballistic phonons or electrons in a single polarization and is given by $\pi^{2} k_{B}^{2} T / 3 h$, where $k_{B}$ and $h$ are the respective Boltzmann and Planck constants, and $T$ is the temperature [1-5]. This minimal and universal amount of heat, for a given $T$, holds for both electrons and phonons, as was theoretically predicted [6-9] and experimentally validated $[9,10]$. Given that the typical mean free paths of electrons and phonons are smaller than $1 \mu \mathrm{m}$ at room temperature, with lower temperatures leading to longer mean free paths, the observation of this quantization in the ballistic regime typically requires the utilization of nanostructures at temperatures lower than $1 \mathrm{~K}$ [10].

The physics of two-dimensional (2D) systems has also become an increasingly important research field due to the fundamental phenomena that have provided the basis for modern device concepts [11, 12]. For instance, a 2D electron gas is typically found in transistor-like structures made from semiconductors that control the mainstream information technologies [13-15]. In addition, with the recent advent of $2 \mathrm{D}$ materials, it is now possible to manipulate $2 \mathrm{D}$ phonon gases and excite fundamental modes of heat transport $[16,17]$. As in the case of $1 \mathrm{D}$ heat conduction driven by either electrons or phonons, these $2 \mathrm{D}$ ballistic modes appear at very low temperatures, which limits their observation and application. However, ballistic heat transport can be achieved at room temperature by surface phonon-polaritons (SPhPs), which are evanescent electromagnetic waves generated by the hybridization of photons and phonons at the interface of polar materials $[18,19]$. This ballistic behavior appears due to the huge SPhP propagation length that was found to be as long as $1 \mathrm{~m}[20,21]$ and hence orders of magnitude longer than the typical mean free paths of electrons and phonons at room temperature. The wavelength of SPhPs propagating along thin films is usually much larger than the film thickness [20], which makes of these energy carriers a $2 \mathrm{D}$ quantum gas. As a result of these relatively long values of the propagation length and wavelength of SPhPs, their contribution to the in-plane heat transport was measured to be comparable to or even higher than the one of phonons [22, 23]. Similar to the case of phonons and electrons, as well as to SPhPs propagating along nanowires [21], the ballistic heat transport of $\mathrm{SPhPs}$ along nanofilms is expected to be characterized by a $2 \mathrm{D}$ quantum of thermal conductance, however its existence and value have not been explored yet. The temperature scaling of this $2 \mathrm{D}$ quantization is expected to be different than that in 1D systems, due to the distinctive features of the density of states.

The purpose of this letter is to demonstrate that the thermal conductance per unit width of a thin enough polar nanofilm supporting the propagation of SPhPs along its surfaces is quantized for any temperature comparable to or lower than room temperature. As the film thickness $d$ decreases, this quantization arises from the convergence of the dispersion relation of SPhPs to that of light in vacuum, while their propagation length $\left(\propto d^{-2}\right)$ increases, which ensures the ballistic propagation of SPhPs in a wide frequency spectrum. This $2 \mathrm{D}$ quantum of thermal conductance appears in thin films of SiN thinner than 50 $\mathrm{nm}$ and therefore its observation could be achieved with the current experimental capabilities.

Let us consider a polar nanofilm supporting the simultaneous propagation of SPhPs and phonons due to the temperature difference $T_{h}>T_{c}$ set by two thermal baths, as shown in Fig. 1. The resulting steady-state heat transport along the $z$ axis is thus driven by the heat fluxes generated by these two types of energy carriers. Assuming that the phonon heat conduction can be described by an effective thermal conductivity $k_{p h}$, the principle of energy conservation along with the Fourier's law establishes that the temperature $T$ inside the nanofilm is given 


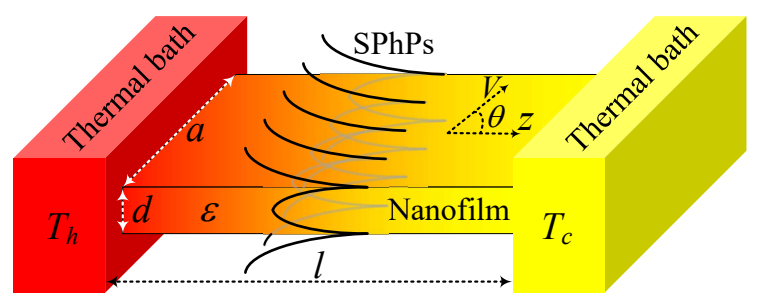

FIG. 1: Scheme of a polar nanofilm supporting the propagation of SPhPs due to the temperature difference $T_{h}-T_{c}>0$ imposed by two thermal baths.

by

$$
-k_{p h} \frac{d T}{d z}+q(z)=q_{t}
$$

where $q$ is the SPhP heat flux and $q_{t}$ is the total heat flux, a constant independent of the position $z$. The phonon thermal conductivity $k_{p h}$ includes the contributions of both the acoustic phonon modes, which usually dominate the heat transport, and the optical ones that couple with photons to generate the SPhPs. Taking into account that SPhPs can be treated like bosonic particles [24], $q$ can be determined by means of the Boltzmann transport equation (BTE) under the relaxation time approximation and the intensity representation. For the steady-state heat transport along the $z$ axis shown in Fig. 1, the BTE takes the form

$$
I_{\omega}+\mu \Lambda \frac{\partial I_{\omega}}{\partial z}=I_{\omega}^{0}
$$

where $\mu=\cos (\theta)$ is the cosine of the angle between the $\mathrm{SPhP}$ propagation direction and the $+z$ axis, $\Lambda=\Lambda(\omega)$ is the propagation length of $\mathrm{SPhPs}, I_{\omega}$ is their intensity, and $I_{\omega}^{0}=V \hbar \omega f_{0}(T) D(\omega)$ its equilibrium counterpart, with $2 \pi \hbar, f_{0}(T)$, and $D(\omega)$ being the Planck constant, Bose-Einstein distribution function and $\mathrm{SPhP}$ density of states per unit area, frequency, and angle; respectively. Since $\mathrm{SPhPs}$ propagate along the interface of the film and they span over its surface, their $2 \mathrm{D}$ density of states is given by[24, 25] $D(\omega)=\beta_{R} /\left((2 \pi)^{2} V\right)$, where $\beta_{R}$ is the real part of the in-plane SPhP wave vector. After solving Eq. (2) for the intensity $I_{\omega}$, the SPhP heat flux can be determined by

$$
q=\frac{1}{d} \iint I_{\omega} \mu d \omega d \theta .
$$

Equation (3) is almost identical to the usual 3D expression for the phonon heat flux, except by its integration on $d \theta$ instead of $d \mu$, due to the 2D heat transport of SPhPs. Once $I_{\omega}$ and $q$ are determined either numerically (accurate method) or analytically (approximate solution), as detailed in the supplementary material (SM) [26], the temperature profile $T(z)$ and total heat flux $q_{t}$ can be determined by integrating Eq. (1) and evaluating its result with the boundary conditions $T(0)=T_{h}$ and $T(l)=T_{c}$. In the very thin film limit of interest in this work, the $\mathrm{SPhP}$ heat flux can be described by the approximate analytical solution of Eqs. (2) and (3), which yield [26]

$$
q(\xi)=\int \frac{\Delta I_{\omega}^{0}}{\lambda d}\left[\pi-2\left(E_{4}(\xi)+E_{4}(\lambda-\xi)\right)\right] d \omega,
$$

where $\xi=z / \Lambda, \lambda=l / \Lambda, \Delta I_{\omega}^{0}=I_{\omega}^{0}\left(T_{h}\right)-I_{\omega}^{0}\left(T_{c}\right)$, and $E_{n}(x)=\int_{0}^{\pi / 2} \mu^{n-2} e^{-x / \mu} d \theta$ is a positive function with values between zero and unity for any $n=1,2, \ldots$. Note that the $\mathrm{SPhP}$ heat flux at two equidistant positions from the external film surfaces $(\xi=0 ; \lambda)$ takes the same value $(q(\xi)=q(\lambda-\xi))$, such that its maximum appears at the middle of the film $(\xi=\lambda / 2)$. This behavior arises from the non-local dependence of the heat flux on the temperature profile [26], which exhibits a non-linear dependence on position, as established by the integration of Eq. (1) for the heat flux in Eq. (4). The explicit results are

$$
\begin{gathered}
T(\xi)=T_{h}-\Delta T \frac{z}{l}+\frac{\Delta T}{k_{p h}} \int k_{\omega}\left[\frac{\pi \xi+2 \Delta \psi(\xi)}{\pi \lambda-4 \psi(0)}-\frac{\xi}{\lambda}\right] d \omega, \\
q_{t}=\left(k_{p o l}+k_{p h}\right) \frac{\Delta T}{l},
\end{gathered}
$$

where $\Delta T=T_{h}-T_{c}, \Delta \psi(\xi)=\psi(\xi)-\psi(0), \psi(x)=$ $E_{5}(x)-E_{5}(\lambda-x)$ and the spectral SPhP thermal conductivity $k_{\omega}$ is defined in terms of its integrated counterpart $k_{p o l}=\int k_{\omega} d \omega$ given by

$$
k_{p o l}=\frac{1}{4 \pi d} \int\left(1-\frac{4 \psi(0)}{\pi \lambda}\right) \hbar \omega \beta_{R} \Lambda \frac{\partial f_{0}}{\partial T} d \omega .
$$

Equation (6) was derived by considering that the average temperature $T=\left(T_{h}+T_{c}\right) / 2 \gg \Delta T$, such that $f_{0}\left(T_{h}\right)-f_{0}\left(T_{c}\right)=\Delta T \partial f_{0} / \partial T$. The rigorous solution of SPhP BTE developed in this work considers the boundary scattering more accurately than the semi-empirical Matthiessen's rule and hence the predictions of $k_{p o l}$ in Eq. (6) are expected to be more precise than those based on this latter rule, as detailed in the Supplementary Material [26]. According to Eq. (5a), the deviation of the temperature profile from the usual linear dependence (first two terms) on position is driven by the ratio $k_{p o l} / k_{p h}$ between the $\mathrm{SPhP}$ and phonon thermal conductivities. Interestingly, regardless of the values of this ratio, the $\mathrm{SPhP}$ contribution to the temperature profile disappears at the middle of the film $(\xi=\lambda / 2)$. This behavior is related to the symmetry of the SPhP heat flux around this position and is well confirmed by accurate numerical results, as shown below. As a result of this symmetry, the sum of temperatures at two equidistant points from the external film surfaces is an invariant of heat conduction given by $T(\xi)+T(\lambda-\xi)=T_{h}-T_{c}$, as established by Eq. (5a). This feature of temperature is generated by the 
non-local behavior of the heat conduction and is analogous to the characteristic temperature profiles found in radiative heat transfer [27].

As the heat transport in a polar nanofilm is driven by both phonons and SPhPs, the total heat flux is determined by the sum of thermal conductivities related to these two energy carriers, as established by Eq. (5b). This fact indicates that, as the film thickness scaled down, the usual reduction of $k_{p h}$ could be offset by the increasing values of $k_{p o l}$, due to the predominant surface effects driving the propagation of SPhPs. In the $\mathrm{SPhP}$ diffusive approximation $(\lambda=l / \Lambda \gg 1)$, the ratio $\psi(0) / \lambda \rightarrow 0$ and Eq. (6) reduces to the previous expression derived by Chen et al. [24], as expected. In the ballistic limit $(\lambda \ll 1)$, on the other hand, $1-4 \psi(0) / \pi \lambda \approx 2 \lambda / \pi$ and the SPhP thermal conductance $G=a d k_{p o l} / l$ of the nanofilm is given by

$$
G=\frac{a \sqrt{\varepsilon_{0}}}{2 \pi^{2} c} \int \hbar \omega^{2} \frac{\partial f_{0}}{\partial T} d \omega
$$

where $c$ is the speed of light in vacuum and $\varepsilon_{0}(=1$ for air) is the relative permittivity of the medium surrounding the nanofilm. Equation (7) was derived by using the fact that the ballistic regime characterized by long propagation lengths $(\Lambda \gg l)$ appears for very thin films along which the SPhPs propagate with a wave vector $\beta_{R}=\sqrt{\varepsilon_{0}} \omega / c$ independent of the film properties $[19,20,24]$. By contrast, the SPhP propagation length for this photon-like mode does depend on the material properties and is generally non-zero in some discrete frequency intervals $[22,23,28] \omega_{n}^{\min } \leq \omega \leq \omega_{n}^{\max }$, with $\omega_{n}^{\min }$ and $\omega_{n}^{\max }$ being the minimum and maximum frequencies supporting the $\mathrm{SPhP}$ propagation for the segment $n=1,2, \ldots, N$. The integration in Eq. (7) for this allowed frequency intervals can then be written as follows

$$
G=\frac{2 a \sqrt{\varepsilon_{0}} k_{B}^{3} T^{2}}{c h^{2}} \sum_{n=1}^{N} \int_{A_{n} / T}^{B_{n} / T} \frac{x^{3} e^{x}}{\left(e^{x}-1\right)^{2}} d x
$$

where $A_{n}=\hbar \omega_{n}^{\min } / k_{B}$ and $B_{n}=\hbar \omega_{n}^{\max } / k_{B}$. The $\mathrm{SPhP}$ thermal conductance is thus determined by the relative values of these normalized cutoff frequencies, characteristic of the film material, with respect to temperature. The analytical calculation of the integral in Eq. (8) yields

$$
\begin{gathered}
G=\frac{2 a \sqrt{\varepsilon_{0}} k_{B}^{3} T^{2}}{c h^{2}} \sum_{n=1}^{N}\left[J\left(A_{n} / T\right)-J\left(B_{n} / T\right)\right], \\
J(x)=\frac{x^{3}}{e^{x}-1}-3 x^{2} \ln \left(1-e^{-x}\right)+6 x L_{2}\left(e^{-x}\right)+6 L_{3}\left(e^{-x}\right)
\end{gathered}
$$

where $L_{s}(z)=\sum_{m=1}^{\infty} \frac{z^{m}}{m^{s}}$ is the polylogarithm function. Taking into account the behavior of the positive function $J$ for small $\left[J(x \ll 1) \approx 6 z(3)-0.5 x^{2}\right]$ and large $\left[J(x \gg 1) \approx\left(x^{3}+3 x^{2}+6 x+6\right) e^{-x}\right]$ arguments, Eq. (9a) establishes that if, for a given temperature $T$, there exists a frequency interval large enough $\left(A_{n} \ll T \ll B_{n}\right)$, the $\mathrm{SPhP}$ thermal conductance per unit width of a nanofilm suspended in air $\left(\varepsilon_{0}=1\right)$ is quantized and given by

$$
\frac{G_{0}}{a}=12 z(3) \frac{k_{B}^{3} T^{2}}{c h^{2}},
$$

where $z(3)=\sum_{m=1}^{\infty} m^{-3} \approx 1.2021$ is the Riemann zeta function. The maximum contribution of SPhPs to the nanofilm thermal conductance is thus independent of the material properties and determined by universal constants only. $G_{0}$ increases with the square of temperature due to the 2D density of states $\left(D(\omega) \propto \beta_{R} \propto \omega\right)$ of SPhPs propagating along a nanofilm, as indicated in Eq.(7). This quadratic increase is thus faster than the linear one exhibited by the ballistic thermal conductance of SPhPs [21], phonons [2, 3, 10], and electrons [9, 29] propagating in a $1 \mathrm{D}$ nanowire. The quantization of the $\mathrm{SPhP}$ thermal condutance of nanofilms supporting the ballistic propagation of SPhPs is analogous to its phonon and electron counterparts observed for $2 \mathrm{D}$ structures at low temperatures $(T<10 \mathrm{~K})$, in which the temperature dependence is generally different than $T^{2}$, due to the non-linear dispersion relations [16, 17, 30].

Note that if the temperature rise $\Delta T=T-T_{0}$ is much smaller than room temperature $\left(\Delta T \ll T_{0}\right)$, $T^{2} \approx T_{0}^{2}+2 T_{0} \Delta T$ and hence $k_{p o l}$ in Eq. (6) increases linearly with $\Delta T$. This linear increase of the SPhP thermal conductivity is consistent with the one observed for the thinnest $(30 \mathrm{~nm}) \mathrm{SiN}$ nanofilm studied in our previous work [31], for temperatures between 300 and $400 \mathrm{~K}$. However, for higher temperatures or thicker films with a stronger phonon heat transport, the temperature dependence of $k_{p o l}$ will be nonlinear and different than that of the measured thermal conductivity [31], because this latter one represents the total (phonon+polariton) thermal conductivity.
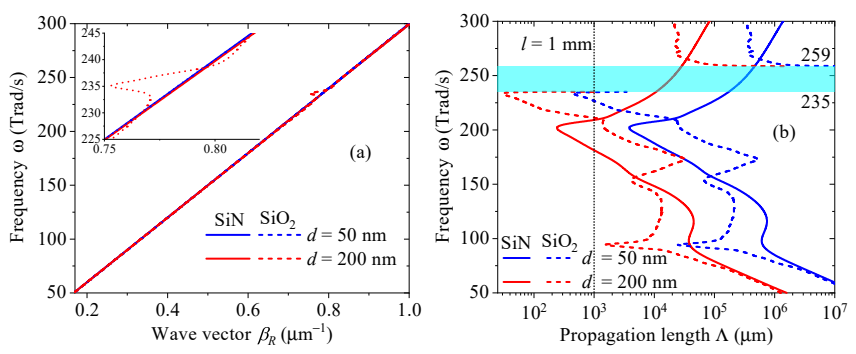

FIG. 2: Frequency spectra of the (a) wave vector and (b) propagation length of SPhPs propagating along ,nanofilms of $\mathrm{SiN}$ and $\mathrm{SiO}_{2}$ of two thicknesses.

Calculations were done with the SPhP dispersion relation[20, 24] for thin films suspended in air and the material permittivities reported in the literature [28]. The light blue region stands for the band bap of $\mathrm{SiO}_{2}$.

To analyze the frequency spectrum supporting the 
propagation of SPhPs, the frequency dependence of the wave vector $\left(\beta_{R}\right)$ and propagation length $(\Lambda)$ of SPhPs propagating along the surfaces of nanofilms of $\mathrm{SiN}$ and $\mathrm{SiO}_{2}$ suspended in air are shown in Figs. 2(a) and 2(b), respectively. In both of these typical polar materials, $\beta_{R}$ increases linearly with frequency through values practically equal to those of the light line $(\omega / c)$, specially for the $50 \mathrm{~nm}$-thick films. The slight deviations from this photon-like behavior characterized by a group velocity close to that of light in vacuum $(V \rightarrow c)$, show up for the $200 \mathrm{~nm}$-thick films due to the increased energy absorption by the material. The relatively weak absorption of thinner films enables SPhPs to propagate distances as long as $1 \mathrm{~m}$, as shown in Fig. 2(b). The peaks (dips) of $\Lambda$ are related to the minima (maxima) of the energy absorption driven by the imaginary part of the film permittivity $[20,28]$. These $\Lambda$ values are comparable to the experimental ones reported for $\mathrm{SiO}_{2}[32]$ and longer than those measured for hBN [33]. Considering that as the film thickness $d$ decreases, the energy absorption reduces and the coupling of SPhPs propagating along both surfaces of the thin film strengthens, the $\mathrm{SPhP}$ propagation length scales as $\Lambda \propto \operatorname{Im}(\varepsilon)^{-1} d^{-2}$ [20, 23], where $\operatorname{Im}(\varepsilon)$ is the imaginary part of the film permittivity that drives the energy absorption by the material from the electromagnetic field. This fact along with the photon-like nature of $\operatorname{SPhPs}\left(\beta_{R} \approx \omega / c\right)$ explains their relatively long propagation length compared to that of phonons and electrons. Note that the SiN nanofilms support the propagation of $\mathrm{SPhPs}$ for all displayed frequencies, while the $\mathrm{SiO}_{2}$ ones have a forbidden frequency band $(235 ; 259) \mathrm{Trad} / \mathrm{s}$, in which $\mathrm{SPhPs}$ do not propagate [28]. In addition, the fact that $\Lambda \gg l=1 \mathrm{~mm}$, for the $50 \mathrm{~nm}$-thick films, indicates that the SPhPs propagate ballistically along them, which is a condition to reach the $2 \mathrm{D}$ quantum of thermal conductance in Eq. (10).
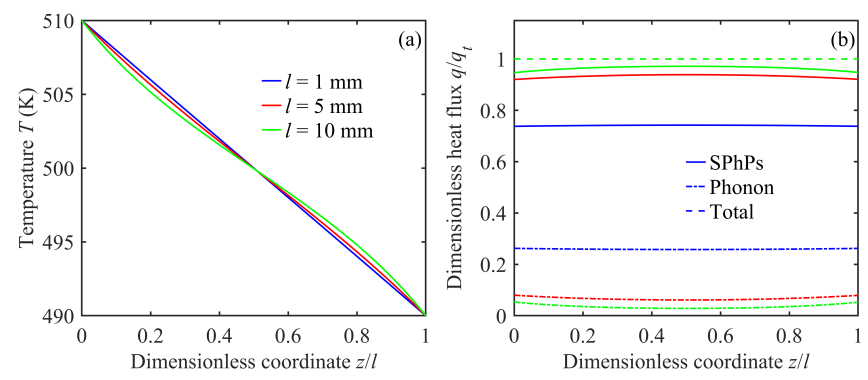

FIG. 3: Numerical results for the (a) temperature profile and (b) heat fluxes in a $50 \mathrm{~nm}$-thick SiN film at $500 \mathrm{~K}$. Calculations were done for three film lengths of practical interest.

Figures 3(a) and 3(b) show the respective temperature and heat flux profiles predicted by the numerical solution of the BTE for the simultaneous propagation of SPhPs and phonon in a $50 \mathrm{~nm}$-thick SiN film with an average temperature of $500 \mathrm{~K}$. For the shortest film, $T$ approaches to the linear profile predicted by the Fourier's heat diffusion equation without heat source, as a result of the ballistic propagation of $\operatorname{SPhPs}(\Lambda \gg l=1 \mathrm{~mm})$ with very weak absorption and negligible energy exchange with phonons. The heat generation rate by $\mathrm{SPhPs}$ is thus small and their contribution to the heat flux is pretty much independent of position, as shown in Fig. 3(b). For longer films with a length $(l=5$ and $10 \mathrm{~mm})$ comparable to the $\mathrm{SPhP}$ propagation length, on the other hand, the quasi-ballistic propagation of SPhPs fosters their energy exchange with phonons, which generates a nonlinear temperature profile similar to that predicted by the heat diffusion equation with a heat sink for $z / l \leq 0.5$ and a heat source for $z / l \geq 0.5$, as seen in Fig. 3(a). The apparent heat sink and heat source terms in the nanofilm arise from the predominant emission and adsorption of SPhPs near its hot and cold sides, respectively. Therefore, the $\mathrm{SPhP}$ heat flux increases with position until $z / l=0.5$ and decreases afterwards, while the phonon counterpart shows the opposite trend, as established by the principle of energy conservation (Eq. (1)). The increase of the $\mathrm{SPhP}$ heat flux with the nanofilm length provides a pathway to enhance the in-plane heat transport along polar films by means of the combined dynamics of SPhPs and phonons, which has never been reported, to the best of our knowledge. Furthermore, as the nonlinearity of the temperature profile represents the fingerprints of SPhPs, its experimental observation can give a conclusive and easy way to detect the SPhP heat transport. For instance, for the $10 \mathrm{~mm}$-long SiN nanofilm shown in Fig. $3(\mathrm{a})$, the largest temperature deviation from the linear profile is about $1 \mathrm{~K}$, which is measurable by the current state-of-the-art infrared thermometers.

The SPhP thermal conductance per unit width $G / a$ of $\mathrm{SiN}$ and $\mathrm{SiO}_{2}$ nanofilms is comparatively shown in Fig. 4 , as a function of the film thickness. As a result of the predominance of surface effects, thinner films exhibit a higher $G / a$, whose values increase with temperature. The analytical (solid lines) and numerical (dashed lines) predictions exhibit a very good agreement for both materials and three temperatures, which confirms the high accuracy of Eq. (6) for quantifying the thermal conductance of SPhPs with ballistic propagation (Fig. 2(b)). More importantly, the upper bounds of $G / a$, in the ballistic regime $(d<50 \mathrm{~nm})$, are well confirmed by the analytical and numerical approaches and their values for the SiN films coincide with the quantum of thermal conductance in Eq. (10), for the three considered temperatures. This is due to the fact that SiN is able to support the propagation of SPhPs in a single branch (Fig. 2) spanning from a very low frequency $\left(A_{1} \rightarrow 0\right)$ to a high one $\omega_{n}^{\max }>300 \mathrm{Trad} / \mathrm{s}\left(B_{1}>2283 \mathrm{~K}\right)$. This fact ensures the $\mathrm{SPhP}$ energy transport over the full frequency spectrum relevant for temperatures comparable to or smaller than room temperature. By contrast, the frequency band gap 
of $\mathrm{SiO}_{2}$ (Fig. 2(b)) reduces this spectrum and therefore the ballistic SPhP thermal conductance to values slightly smaller than the quantum of thermal conductance, as shown in Fig. 4. This quantization can thus only be achieved in nanofilms without frequency band gaps for the propagation of SPhPs and could be observed by using an experimental setup sensitive to the $\mathrm{SPhPs}$ energy transport [34].

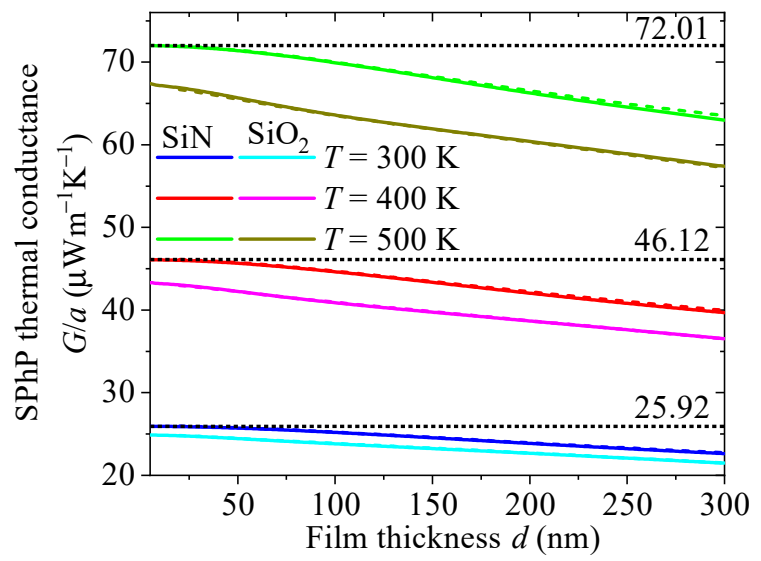

FIG. 4: SPhP thermal conductance per unit width of 1 mm-long nanofilms of $\mathrm{SiN}$ and $\mathrm{SiO}_{2}$, as a function of their thickness. The solid and dashed lines represent the respective analytical (Eq. (6)) and numerical [26] predictions, while the dotted ones stand for the quantum of thermal conductance predicted by Eq. (10). Calculations were done for three temperatures.

In summary, the existence of the universal quantum of thermal conductance $12 z(3) k_{B}^{3} T^{2} / c h^{2}$ per unit width of a thin enough polar nanofilm supporting the propagation of SPhPs along its surfaces has been demonstrated for temperatures comparable to or lower than room temperature. This quantization arises from the ballistic behavior of SPhPs and represents the upper bound for their heat transport, which in $\mathrm{SiN}$, is reached for a film thinner than $50 \mathrm{~nm}$. The obtained results for the SPhP thermal conductance and SPhP thermal conductivity as well as for the temperature and heat flux profiles driven by the combined dynamics of phonons and SPhPs, thus quantify the potential of the latter heat carriers to improve the thermal performance of nanomaterials and facilitate its observation, as these four parameters are measurable.

This work was supported by the Postdoctoral Fellowship of Japan Society for the Promotion of Science, grant $n^{\circ}$ P19353, CREST Japan Science and Technology Agency, grants $n^{\circ}$ JPMJCR19Q3 and JPMJCR19I1, and French project ANR-19-CE09-0005 "EPolariton".

* ordonez@iis.u-tokyo.ac.jp
[1] B. A. Glavin, Low-temperature heat transfer in nanowires, Phys. Rev. Lett. 86, 4318 (2001).

[2] L. G. C. Rego and G. Kirczenow, Quantized thermal conductance of dielectric quantum wires, Phys. Rev. Lett. 81, 232 (1998).

[3] T. Yamamoto, S. Watanabe, and K. Watanabe, Universal features of quantized thermal conductance of carbon nanotubes, Phys. Rev. Lett. 92, 075502 (2004).

[4] Y. Chalopin, J.-N. Gillet, and S. Volz, Predominance of thermal contact resistance in a silicon nanowire on a planar substrate, Phys. Rev. B 77, 233309 (2008).

[5] R. Venkatesh, J. Amrit, Y. Chalopin, and S. Volz, Thermal resistance of metal nanowire junctions in the ballistic régime, Phys. Rev. B 83, 115425 (2011).

[6] M. Büttiker, Four-terminal phase-coherent conductance, Phys. Rev. Lett. 57, 1761 (1986).

[7] R. Landauer, Spatial variation of currents and fields due to localized scatterers in metallic conduction, IBM J. Res. Dev. 1, 223 (1957).

[8] R. Landauer, Can a length of perfect conductor have a resistance, Phys. Lett. A 85, 91 (1981).

[9] O. Chiatti, J. T. Nicholls, Y. Y. Proskuryakov, N. Lumpkin, I. Farrer, and D. A. Ritchie, Quantum thermal conductance of electrons in a one-dimensional wire, Phys. Rev. Lett. 97, 056601 (2006).

[10] K. Schwab, E. Henriksen, J. Worlock, and M. Roukes, Measurement of the quantum of thermal conductance, Nature 404, 974 (2000).

[11] A. F. Santander-Syro, O. Copie, T. Kondo, F. Fortuna, S. Pailhès, R. Weht, X. G. Qiu, F. Bertran, A. Nicolaou, A. Taleb-Ibrahimi, P. L. Fèvre, G. Herranz, M. Bibes, N. Reyren, Y. Apertet, P. Lecoeur, A. Barthélémy, and M. J. Rozenberg, Two-dimensional electron gas with universal subbands at the surface of srtio $_{3}$, Nature 469, 189 (2011).

[12] K. S. Novoselov, V. I. Falko, L. Colombo, P. R. Gellert, M. G. Schwab, and K. Kim, A roadmap for graphene, Nature 490, 192 (2012).

[13] J. R. Prance, C. G. Smith, J. P. Griffiths, S. J. Chorley, D. Anderson, G. A. C. Jones, I. Farrer, and D. A. Ritchie, Electronic refrigeration of a two-dimensional electron gas, Phys. Rev. Lett. 102, 146602 (2009).

[14] G. F. Giuliani and J. J. Quinn, Lifetime of a quasiparticle in a two-dimensional electron gas, Phys. Rev. B 26, 4421 (1982).

[15] B. Tanatar and D. M. Ceperley, Ground state of the twodimensional electron gas, Phys. Rev. B 39, 5005 (1989).

[16] K. Saito, J. Nakamura, and A. Natori, Ballistic thermal conductance of a graphene sheet, Phys. Rev. B 76, 115409 (2007).

[17] M.-H. Bae, Z. Li, Z. Aksamija, P. N. Martin, F. Xiong, Z.-Y. Ong, I. Knezevic, and E. Pop, Ballistic to diffusive crossover of heat flow in graphene ribbons, Nat. Commun. 4, 1734 (2013).

[18] V. M. Agranovich, Surface Polaritons (Elsevier, Amsterdam, 2012).

[19] F. Yang, J. R. Sambles, and G. W. Bradberry, Longrange surface modes supported by thin films, Phys. Rev. B 44, 5855 (1991).

[20] J. Ordonez-Miranda, L. Tranchant, T. Tokunaga, B. Kim, B. Palpant, Y. Chalopin, T. Antoni, and S. Volz, Anomalous thermal conductivity by surface phononpolaritons of polar nano thin films due to their asymmetric surrounding media, J. Appl. Phys. 113, 084311 
(2013).

[21] J. Ordonez-Miranda, L. Tranchant, B. Kim, Y. Chalopin, T. Antoni, and S. Volz, Quantized thermal conductance of nanowires at room temperature due to zenneck surface-phonon polaritons, Phys. Rev. Lett. 112, 055901 (2014).

[22] L. Tranchant, S. Hamamura, J. Ordonez-Miranda, T. Yabuki, A. Vega-Flick, F. Cervantes-Alvarez, J. J. Alvarado-Gil, S. Volz, and K. Miyazaki, Two-dimensional phonon polariton heat transport, Nano Lett. 19, 6924-6930 (2019).

[23] Y. Wu, J. Ordonez-Miranda, S. Gluchko, R. Anufriev, D. D. S. Meneses, L. D. Campo, S. Volz, and M. Nomura, Enhanced thermal conduction by surface phononpolaritons, Sci. Adv. 6, eabb4461 (2020).

[24] D.-Z. A. Chen, A. Narayanaswamy, and G. Chen, Surface phonon-polariton mediated thermal conductivity enhancement of amorphous thin films, Phys. Rev. B 72, 155435 (2005).

[25] I. Dorofeyev, On the equilibrium spectrum of surface polaritons thermally excited by solids, Phys. Lett. A 375, 2885 (2011).

[26] S. S. M. at xyz for the detailed procedures to find the analytical and numerical solutions., .

[27] V. Kourganoff, Basic Methods in Transfer Problems (Dover Publications, New York, 1963).

[28] J. Ordonez-Miranda, S. Volz, and M. Nomura, Surface phonon-polariton heat capacity of polar nanofilms, Phys.
Rev. Applied 15, 054068 (2021).

[29] R. Venkatesh, J. Amrit, Y. Chalopin, and S. Volz, Thermal resistance of metal nanowire junctions in the ballistic regime, Phys. Rev. B 83, 115425 (2011).

[30] N. Mingo and D. A. Broido, Carbon nanotube ballistic thermal conductance and its limits, Phys. Rev. Lett. 95, 096105 (2005).

[31] Y. Wu, J. Ordonez-Miranda, L. Jalabert, S. Tachikawa, R. Anufriev, H. Fujita, S. Volz, and M. Nomura, Observation of quasi-ballistic thermal transport of surface phonon-polaritons over hundreds of micrometres (2021), arXiv:2107.06391.

[32] S. Gluchko, B. Palpant, S. Volz, R. Braive, and T. Antoni, Thermal excitation of broadband and long-range surface waves on sio2 submicron films, Appl. Phys. Lett. 110, 263108 (2017).

[33] A. J. Giles, S. Dai, I. Vurgaftman, T. Hoffman, S. Liu, L. Lindsay, C. T. Ellis, N. Assefa, I. Chatzakis, T. L. Reinecke, J. G. Tischler, M. M. Fogler, J. H. Edgar, D. N. Basov, and J. D. Caldwell, Ultralow-loss polaritons in isotopically pure boron nitride, Nature Mater. 17, 134 (2018).

[34] S. Shin, M. Elzouka, R. Prasher, and R. Chen, Far-field coherent thermal emission from polaritonic resonance in individual anisotropic nanoribbons, Nat. Commun. 10, 1377 (2019). 